\title{
Crack detection based on optimal control
}

\author{
B. Chomette ${ }^{a, b}$ and J-J. Sinou ${ }^{a}$ \\ ${ }^{a}$ Laboratoire de Tribologie et Dynamique des Systèmes UMR-CNRS 5513 \\ École Centrale de Lyon, 36 avenue Guy de Collongue \\ 69134 Ecully Cedex, France \\ ${ }^{b}$ Institut d'Alembert UMR-CNRS 7190 \\ Université Pierre et Marie Curie, 4 place Jussieu \\ 75252 Paris Cedex 05, France
}

\begin{abstract}
Techniques for optimal control to increase system security attract a great deal of interest from industry. The presence of transversal cracks can considerably modify the dynamics of a system. In the case of closed-loop systems, i.e. controlled systems, these faults can cause the destabilization and variation of control performance. Consequently, such variations can be used to detect transversal cracks. Therefore the present study proposes an investigation into the possibility of detecting structure modification based on estimated control performance by using the Rational Fraction Polynomial (RFP) algorithm. This method is applied numerically to a multi-cracked controlled truss. Both the optimal control of the cracked structure and the possibility of detecting the presence of cracks by monitoring the evolution of control performance are studied. The efficiency of the proposed method is demonstrated through numerical simulations corresponding to different crack orientations and locations.
\end{abstract}

\section{Introduction}

Cracks and thus structural modifications cause robustness problems, particularly reduced performance, in structures controlled by modal control. One way to detect cracks is to use modal testing in which changes in modal parameters such as variations in frequencies and mode shapes are used to detect damage. A review based on the detection of structural damage through changes in frequencies was discussed by Salawu (1997). Moreover, Dilena and Morassi (2002) proposed damage identification based on changes in the nodes of mode shapes. They demonstrated that appropriate use of resonances and antiresonances can be used to avoid the non-uniqueness of damage location for symmetrical beams. One of the limitations of the indexes based on the change in the modal properties of changes in cracked structures is the use of only resonant frequencies and mode shapes for the vibrational description of mechanical structures. This has led to extending damage detection based on the use of the Frequency Response Function. For example, Faverjon and Sinou (2008, 2009) proposed a damage assessment technique based on the constitutive relation error updating method for the non-destructive detection and sizing of single and multiple open cracks in beams. They demonstrated that it is possible to detect the number of cracks in the beam and estimate both crack positions and sizes with satisfactory precision, despite the presence of noise levels 
and the use of a limited number of degrees of freedom for the identification procedure. Comprehensive surveys can be found in the papers of Doebling et al. (1996), Sohn et al. (2003) and Sinou (2009) for structural health monitoring and damage detection techniques in the civil and mechanical engineering communities.

However little effort has been dedicated to discussing the capability of a controlled multi-crack truss structure to detect cracks by using modal parameters and controlling performance. Vibration control, by using optimally located actuators and sensors (Hac and Liu, 1993), is one way of controlling structures and limiting vibration damage (Chomette et al. 2010). Several active control strategies such as active collocated control (Preumont, 2002) or passive strategies (Badel et al., 2006) can be used. Active modal control is a solution for targeting the control energy only on the vibration modes of interest with a minimum number of components (Gaudiller and der Hagopian, 1996). In the case of modal control based on a Luenberger observer (Luenberger, 1971), structural modifications lead to changes in control performance (Chomette et al., 2008), and such changes can be used to detect cracks. Moreover, identification algorithms can be used to obtain structure modal parameters (Richardson and Formenti, 1985) and control variations in performance. Therefore we propose to demonstrate the efficiency of an optimal control and identification procedure for a complex structure with multiple cracks. This study focuses not only on crack depth and position but also crack orientation, which can drastically modify the dynamic behavior of damaged mechanical structures. We will thus show that the evolution of control parameters in relation to other parameters (such as crack orientation) can be early indicators of the presence of a crack in a mechanical structure. The paper is organized as follows. Section 2 summarizes the concept underlying crack modeling and the model of the complete truss structure. The third part deals with the control and identification procedure. Then, control performance variation will be applied as a sensitive indicator of structural integrity. The efficiency of the methodology proposed will be investigated in the last part of the paper through different numerical simulations of a multi-cracked controlled truss with different cracks configurations.

\section{Equation of motion of the system}

In this section, the modeling of a transverse crack is proposed first, after which the equation of motion for the complete system with multi-cracks is presented.

\subsection{Crack model}

The modeling of a transverse crack that was described extensively in (Sinou, 2007) is briefly presented here. Mayes and Davies (1984) demonstrated that a transverse crack in a shaft can be represented by reducing the second moment of area $\Delta I$ of the element at the location of the crack

$$
\frac{\Delta I}{I_{0}}=\frac{\frac{R}{l}\left(1-\nu^{2}\right) F(\mu)}{1+\frac{R}{l}\left(1-\nu^{2}\right) F(\mu)}
$$

for a circular beam. $I_{0}, R, l$, and $\nu$ are the second moment of the area, the shaft radius, the length of the section and the Poisson ratio, respectively. $\mu$ is the non-dimensional crack depth and is defined by $\mu=\frac{h}{R} . F(\mu)$ is the fractional change in the second moment of the area measured at the crack face by considering a series of experimental tests for different transverse cracks. It should be noted that there are two values of $F(\mu)$ for a given transverse crack, one for each of the orthogonal directions in the plane of the crack. The cross section 
of the shaft at the location of the crack has asymmetric area moments of inertia about the neutral axis of bending due to the presence of the crack.

Then, for each orientation $\chi$ of the crack (see Figure 1(a), the crack model is derived by assuming that the portion of the chordal crack lying below the neutral axis will be opened under the influence of self weight bending. As previously proposed by Lees and Friswell (2001), the compliance functions given in Equation 1 are evaluated in the two orthogonal directions by assuming equivalence with the chordal crack of equal effective area. This procedure is sufficient to reflect the main physics of the open and closed portions for a particular crack depth and orientation by assuming beam self-weight, as previously mentioned by Lees and Friswell (2001).

So, the cross-section of the beam at the location of the crack has asymmetric area moments inertia $I_{\eta}$ and $I_{\xi}$ about the centroidal axes $\eta$ and $\xi$ that are defined as

$$
\begin{gathered}
I_{\eta}=\widetilde{I}_{\eta} \\
I_{\xi}=\widetilde{I}_{\xi}-A \bar{X}^{2}
\end{gathered}
$$

The area moments of inertia $\widetilde{I}_{\eta}$ and $\widetilde{I}_{\xi}$ contain the reduced moment of area $\Delta I$ for each of the orthogonal directions $\eta$ and $\xi$, as indicated in Equation 1. The complete expressions are given in (Sinou, 2005 and 2007). $\bar{X}$ defines the new centroid due to the presence of the crack (i.e. $\left.\bar{X}=\frac{2}{3 A} R^{3}\left(2 \mu-\mu^{2}\right)^{\frac{3}{2}}\right)$ and $A$ is the sector area of crack (i.e. $A=$ $\left.R^{2}\left((1-\mu) \sqrt{2 \mu-\mu^{2}}+\cos ^{-1}(1-\mu)\right)\right)$.

Using the standard finite element formulation, the stiffness contribution $\mathbf{K}_{c r a c k}^{\eta, \xi}$ of an open crack about the centroidal axes $\eta$ and $\xi$ for a two nodes Timoshenko beam element of length $l$ and Young's modulus $E$ (corresponding to the flexible degree-of-freedom $\left[u_{1} ; v_{1} ; \theta_{1} ; \psi_{1} ; u_{2} ; v_{2} ; \theta_{2} ; \psi_{2}\right]$, see Figure 1(b) can be defined at the crack location

$$
\mathbf{K}_{\text {crack }}^{\eta, \xi}=\frac{E}{l^{3}}\left(\begin{array}{cccccccc}
12 I_{\eta} & 0 & 0 & 6 l I_{\eta} & -12 I_{\eta} & 0 & 0 & 6 l I_{\eta} \\
& 12 I_{\xi} & -6 l I_{\xi} & 0 & 0 & -12 I_{\xi} & -6 l I_{\xi} & 0 \\
& & 4 l^{2} I_{\xi} & 0 & 0 & 6 l I_{\xi} & 2 l^{2} I_{\xi} & 0 \\
& & & 4 l^{2} I_{\eta} & -6 l I_{\eta} & 0 & 0 & 2 l^{2} I_{\eta} \\
& & & & 12 l I_{\eta} & 0 & 0 & -6 l I_{\eta} \\
& & & & & 12 l I_{\xi} & 6 l I_{\xi} & 0 \\
& & & & & & 4 l^{2} I_{\xi} & 0 \\
& & & & & & & 4 l^{2} I_{\eta}
\end{array}\right)
$$

Finally, the effects of the crack on the axial load terms can be written by superimposing the axial stiffness terms over the bending stiffness.

\subsection{Model of the complete truss structure}

After assembling the different beam elements and the stiffness contribution of the multi-cracks, the equations of motion of the truss structure are given by

$$
\mathbf{M} \ddot{\mathbf{x}}+\mathbf{C} \dot{\mathbf{x}}+\left(\mathbf{K}-\mathbf{K}_{\text {multi-cracks }}\right) \mathbf{x}=\mathbf{F}_{e}
$$

where $\ddot{\mathbf{x}}, \dot{\mathbf{x}}$ and $\mathbf{x}$ are the acceleration, velocity and displacement of the degrees-of-freedom of the cracked system. $\mathbf{M}, \mathbf{C}$ and $\mathbf{K}$ are the mass, damping and stiffness matrices respectively. $\mathbf{F}_{e}$ defines an external force vector (given by $\mathbf{F}_{e}=\mathbf{F} e^{i \omega t}$ where $\mathbf{F}$ defines the force amplitude). $\mathbf{K}_{\text {multi-cracks }}$ is the final stiffness matrix due to the presence of multi-cracks. 


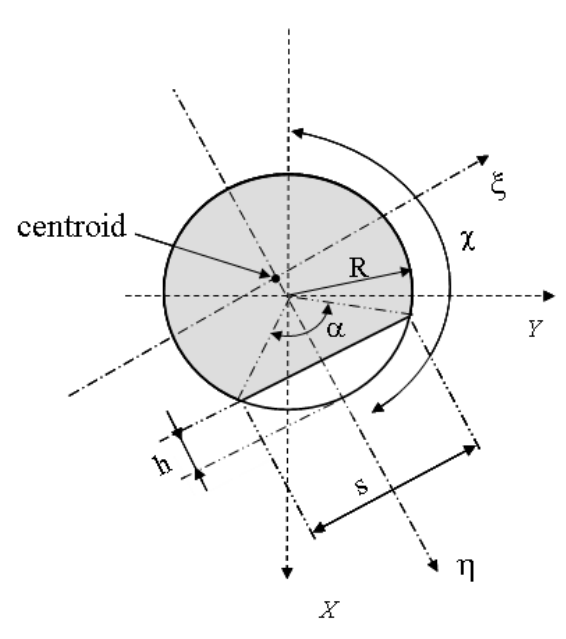

(a) Crack element

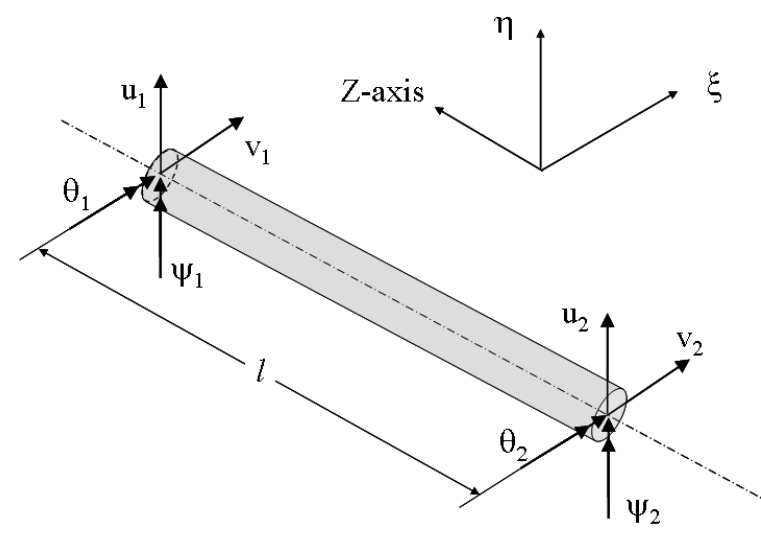

(b) Description of the Dof in $\eta$ and $\xi$-axes

Figure 1: Description of the crack element

\section{Optimal control and identification procedure}

\subsection{Control design}

In this paper, an active modal control based on a Linear Quadratic Gaussian algorithm is applied to the multi-cracked structure in order to demonstrate the multi-cracks detection possibility in the case of controlled structures. Indeed, modal control is based on a model of the structure and its performances depends on the model's precision. To aid understanding, it should be borne in mind that a slight difference between the model and the structure can lead to a decrease in performance or instability in the system controlled. In this short section of the study, this decrease in robustness is investigated to detect cracks caused by a slight variation of modal parameters.

The linear control need to use a linear model which can be described in the state form by

$$
\left\{\begin{array}{l}
\dot{\mathbf{x}}=\mathbf{A x}+\mathbf{B} u+\mathbf{G} w \\
\mathbf{y}=\mathbf{C x}
\end{array}\right.
$$

where $\mathbf{A}$ is the dynamical system matrix, $\mathbf{B}$ and $\mathbf{C}$ are the actuator and sensor matrices respectively and $\mathbf{G}$ is the disturbance matrix. $\mathbf{x}$ and $\mathbf{y}$ are the state and the output vectors respectively. $u$ is the control and $w$ the disturbance. In the case of one actuator and one disturbance point, $u$ and $w$ are scalar quantities. The state matrices can be written for $n$ modes

$$
\begin{gathered}
\mathbf{A}=\left(\begin{array}{cc}
\mathbf{0}_{n, n} & \operatorname{diag}\left(\omega_{i}\right) \\
-\operatorname{diag}\left(\omega_{i}\right) & -\operatorname{diag}\left(2 \xi_{i} \omega_{i}\right)
\end{array}\right) \\
\mathbf{B}=\left(\begin{array}{c}
\mathbf{0}_{n, 1} \\
\boldsymbol{\Pi}^{a}
\end{array}\right) \\
\mathbf{G}=\left(\begin{array}{c}
\mathbf{0}_{1, n} \\
\boldsymbol{\Pi}^{f}
\end{array}\right) \\
\mathbf{C}=\left(\begin{array}{ll}
\boldsymbol{\Pi}^{c} \omega^{-1} & \mathbf{0}_{1, n}
\end{array}\right) \\
\mathbf{x}=\left(\begin{array}{c}
\omega \mathbf{q} \\
\dot{\mathbf{q}}
\end{array}\right)
\end{gathered}
$$


where $\mathbf{q}$ is the modal displacement vector, $\omega_{i}$ and $\xi_{i}$ are the natural frequencies and modal Rayleigh damping respectively. $\Pi^{a}, \Pi^{c}$ and $\Pi^{f}$ are the actuator, sensor and disturbance vectors respectively in the modal basis, defined as

$$
\begin{aligned}
& \Pi^{a}=\phi^{t} \mathbf{K}^{a} \\
& \boldsymbol{\Pi}^{c}=\phi^{t} \mathbf{K}^{c} \\
& \boldsymbol{\Pi}^{f}=\boldsymbol{\phi}^{t} \mathbf{K}^{f}
\end{aligned}
$$

where $\mathbf{K}^{a}, \mathbf{K}^{c}$ and $\mathbf{K}^{f}$ are the actuator, sensor and disturbance vectors of degrees of freedom, respectively, and $\phi$ is the mode shapes matrix. The control $u$ is solution of the Linear Quadratique Regulator problem and is calculated by using the state vector $\mathbf{x}$ and the control gain matrix $\mathbf{K}$

$$
u=-\mathbf{K} \mathbf{x} \text {. }
$$

As modal state vector $\mathbf{x}$ cannot be measured, a Luenberger observer (Luenberger 1971) is used to reconstruct the state vector $\hat{\mathbf{x}}$. The estimated state vector can be written

$$
\left\{\begin{array}{l}
\dot{\hat{\mathbf{x}}}=\mathbf{A}_{m} \hat{\mathbf{x}}+\mathbf{B}_{m} u+\mathbf{L}(\mathbf{y}-\hat{\mathbf{y}}) \\
\hat{\mathbf{y}}=\mathbf{C}_{m} \hat{\mathbf{x}}
\end{array}\right.
$$

where $\left(\mathbf{A}_{m}, \mathbf{B}_{m}, \mathbf{C}_{m}\right)$ is the state model of the Luenberger observer used to reconstruct the state vector. $\mathbf{L}$ is the observer gain calculated by using the Linear Quadratic Gaussien algorithm.

\subsection{Frequency Response Function calculation}

The transfer function of the uncontrolled system can be written for one sensor as

$$
H_{B O}(j \omega)=\frac{y}{w}=\mathbf{C}_{s}\left(j \omega \mathbf{I} \mathbf{d}-\mathbf{A}_{s}\right)^{-1} \mathbf{G}_{s}
$$

where $\left(\mathbf{A}_{s}, \mathbf{B}_{s}, \mathbf{C}_{s}\right)$ is the state model that represents the structure in the numerical simulations. The transfer function of the system controlled can be written for one sensor as

$$
H_{B F}(j w)=\frac{y}{w}=\mathbf{C}_{s}\left(j \omega \mathbf{I} \mathbf{d}-\left(\mathbf{A}_{s}-\mathbf{B}_{s} \mathbf{K}\left(j \omega \mathbf{I d}-\left(\mathbf{A}_{m}-\mathbf{B}_{m} \mathbf{K}-\mathbf{L} \mathbf{C}_{m}\right)\right)^{-1} \mathbf{L C}_{s}\right)\right)^{-1} \mathbf{G}_{s}
$$

The two state models $\left(\mathbf{A}_{s}, \mathbf{B}_{s}, \mathbf{C}_{s}\right)$ and $\left(\mathbf{A}_{m}, \mathbf{B}_{m}, \mathbf{C}_{m}\right)$ are assumed to be equal in the case of an uncracked structure. This configuration will be called the nominal configuration. In the case of a cracked structure, modifications between these two models can lead to modifications in the dynamics of the closed loop system. In this paper, these modifications are estimated by using the RFP algorithm in order to detect the crack. Thus the system $\left(\mathbf{A}_{s}, \mathbf{B}_{s}, \mathbf{C}_{s}\right)$ which represents the structure can be calculated for different crack orientation from $\chi=0^{\circ}$ to $\chi=180^{\circ}$. For example, the numerical transfer function in open and closed loop can be written for $\chi=180^{\circ}$

$$
\begin{gathered}
H_{B O}(j \omega)=\frac{y}{w}=\mathbf{C}_{180^{\circ}}\left(j \omega \mathbf{I d}-\mathbf{A}_{180^{\circ}}\right)^{-1} \mathbf{G}_{180^{\circ}} \\
H_{B F}(j \omega)=\frac{y}{w}=\mathbf{C}_{180^{\circ}}\left(j \omega \mathbf{I d}-\left(\mathbf{A}_{180^{\circ}}-\mathbf{B}_{180^{\circ}} \mathbf{K}\left(j \omega \mathbf{I d}-\left(\mathbf{A}_{m}-\mathbf{B}_{m} \mathbf{K}-\mathbf{L} \mathbf{C}_{m}\right)\right)^{-1} \mathbf{L}_{180^{\circ}}\right)\right)^{-1} \mathbf{G}_{180^{\circ}}
\end{gathered}
$$


where $\left(\mathbf{A}_{180^{\circ}}, \mathbf{B}_{180^{\circ}}, \mathbf{C}_{180^{\circ}}\right)$ is the system which represents the cracked truss with a transversal crack for $\chi=180^{\circ}$. The mode shapes variation is taken into account in the modal matrices $\mathbf{B}_{180^{\circ}}, \mathbf{C}_{180^{\circ}}$ and $\mathbf{G}_{180^{\circ}}$ based on actuator, sensor and disturbance vectors. Indeed actuator, sensor and disturbance vectors are calculated using the cracked mode shapes matrix $\phi_{180^{\circ}}$

$$
\begin{aligned}
& \boldsymbol{\Pi}_{180^{\circ}}^{a}=\phi_{180}{ }^{\circ} \mathbf{K}^{a} \\
& \boldsymbol{\Pi}_{180^{\circ}}^{c}=\phi_{180{ }^{\circ}} \mathbf{K}^{c} \\
& \boldsymbol{\Pi}_{180^{\circ}}^{f}=\phi_{180}{ }^{\circ} \mathbf{K}^{f}
\end{aligned}
$$

\subsection{Optimal placement}

In the case of complex structures, like truss structures, the actuators and sensors must be located optimally in order to control the system. The location of the optimal control component is calculated by using controllability and observability gramians theory. The controllability or observability problem can be linked to the crack detection problem. The best point to detect a crack is a point where all the modes affected by the crack are observable. Consequently, it corresponds to the best point for controlling the structure. The optimal node is obtained from the Hac and Liu (1993) criterion

$$
P I=\sum_{i=1}^{2 m} \lambda_{i} \sqrt[2 m]{\prod_{i=1}^{2 m} \lambda_{i}}
$$

where $\mathrm{m}$ is the mode number used in the computation and $\lambda_{i}$ are the eigenvalues of the Gramian matrix $\mathbf{W}_{c}$ defined as

$$
\mathbf{W}_{c}=\int_{O}^{\infty} e^{\mathbf{A} \tau} \mathbf{B B}^{t} e^{\mathbf{A}^{t} \tau} d \tau
$$

\subsection{Identification procedure}

The aim of the identification procedure is to estimate the variation of the modal parameters. The variation of the modal parameters (i.e. frequency, damping and modal amplitude constant variation), and the control performance variation are estimated by using the Rational Fraction Polynomial (RFP) algorithm (Richardson and Formenti, 1985) applied on the com-

plex Frequency Response Function (FRF) obtained by using the truss model. The estimated FRF can be written as

$$
H(\omega)=\sum_{k=1}^{n} \frac{a_{k}}{\omega_{k}^{2}-\omega^{2}+2 \xi_{k} \omega_{k} \omega}
$$

where $\mathrm{n}$ in the mode number, $\omega_{k}$ and $\xi_{k}$ are respectively the frequencies and modal damping, $a_{k}$ is the modal amplitude constant of the FRF.

\section{Numerical application}

\subsection{The multi-cracked truss studied}

The procedure explained in the previous section is applied numerically to the model of the cracked truss presented in figure 2, The truss is composed of 170 elements and 123 nodes and 
is clamped on nodes 122 and 123. The disturbance is applied on node 111. The Hac and Liu criterion calculated on the truss to optimally locate the controller is shown in figure 3(a) for nodes 1 to 120. The optimal location for the control is located on node 114. This location does not depend on the disturbance point. It means that nodes 111 to 121 (zoom in figure 3(b) are the most sensitive to the behavior of the full truss. This node is chosen to apply the collocated control and to get observation of the vibration. The displacement used by the Luenberger observer to reconstruct the state vector is measured on this node. The control calculated by the LQ controller is then applied on this node. The truss model includes 20 modes and the control is performed by focusing on the first six modes.

In the following study, we focus our attention on modes 1 to 6 of the truss. Identification of the modal parameters (frequency, damping and amplitude) is performed with only one collocated actuator-sensor.

Two cases are studied in order to demonstrate the efficiency method in different configurations. First, one transversal crack is located on element 52 and two straight cracks are located on elements 15 and 39 respectively (with depth $\mu=1$ ). Secondly, one transversal crack is located on element 12 and two straight cracks are located on elements 46 and 29 respectively (with depth $\mu=1$ ). The transversal crack orientation can change from $\chi=0^{\circ}$ to $\chi=180^{\circ}$. When $\chi=0^{\circ}$, the crack is fully open and when $\chi=180^{\circ}$, the crack is totally closed.

As previously explained, it is assumed that the static deflection is much greater that the dynamic response of the cracked system. So, for each orientation of the crack, the crack model is derived by assuming that the portion of the chordal crack lying below the neutral axis will be opened under the influence of self weight bending (Lees and Fiswell, 2001; Sinou, 2005 and 2007).

The identified modal parameters and the mode shapes for the uncracked structure are shown in table 1 and figure 4. For the interested reader, it may be noted that variation of mode shapes are not used in the following sections to detect the presence of cracks.

\begin{tabular}{ccc}
\hline Frequency $[\mathrm{Hz}]$ & Modal damping & Modal amplitude [dB] \\
\hline 141,8 & $9,97 \times 10^{-4}$ & $-55,78$ \\
433,0 & $9,93 \times 10^{-4}$ & $-64,67$ \\
857,0 & $1,57 \times 10^{-3}$ & $-79,09$ \\
1238,1 & $2,28 \times 10^{-3}$ & $-81,48$ \\
1414,1 & $2.53 \times 10^{-3}$ & $-78,74$ \\
1499,8 & $3,20 \times 10^{-3}$ & $-97,99$ \\
\hline
\end{tabular}

Table 1: Modal parameters of the uncracked system

\subsection{Multi-cracked uncontrolled truss}

Firstly, we consider the evolution of the cracked orientation from $\chi=0^{\circ}$ to $\chi=180^{\circ}$ for the transversal crack and the two straight cracks. The effects on the modal parameters are illustrated in figures 5 and 6 for the two cases. The estimation is carried out in the frequency range $0-2500 \mathrm{~Hz}$ with 10 modes. The crack orientation from $\chi=0^{\circ}$ to $\chi=180^{\circ}$ causes a frequency variation $(-8.8 \%$ for the first mode, $-3.1 \%$ for the second mode, $-6.7 \%$ for the third mode, $-1.9 \%$ for the fourth mode, $-4.5 \%$ for the fifth mode and $-3.0 \%$ for the sixth mode in the case $1 ;-8.8 \%$ for the first mode, $-3.1 \%$ for the second mode, $-6.4 \%$ for the third mode, $-2.6 \%$ for the fourth mode, $-4.5 \%$ for the fifth mode and $-3.1 \%$ for the sixth mode in the case 2 ). Consequently, one of the classical results previously reported by certain researchers is 


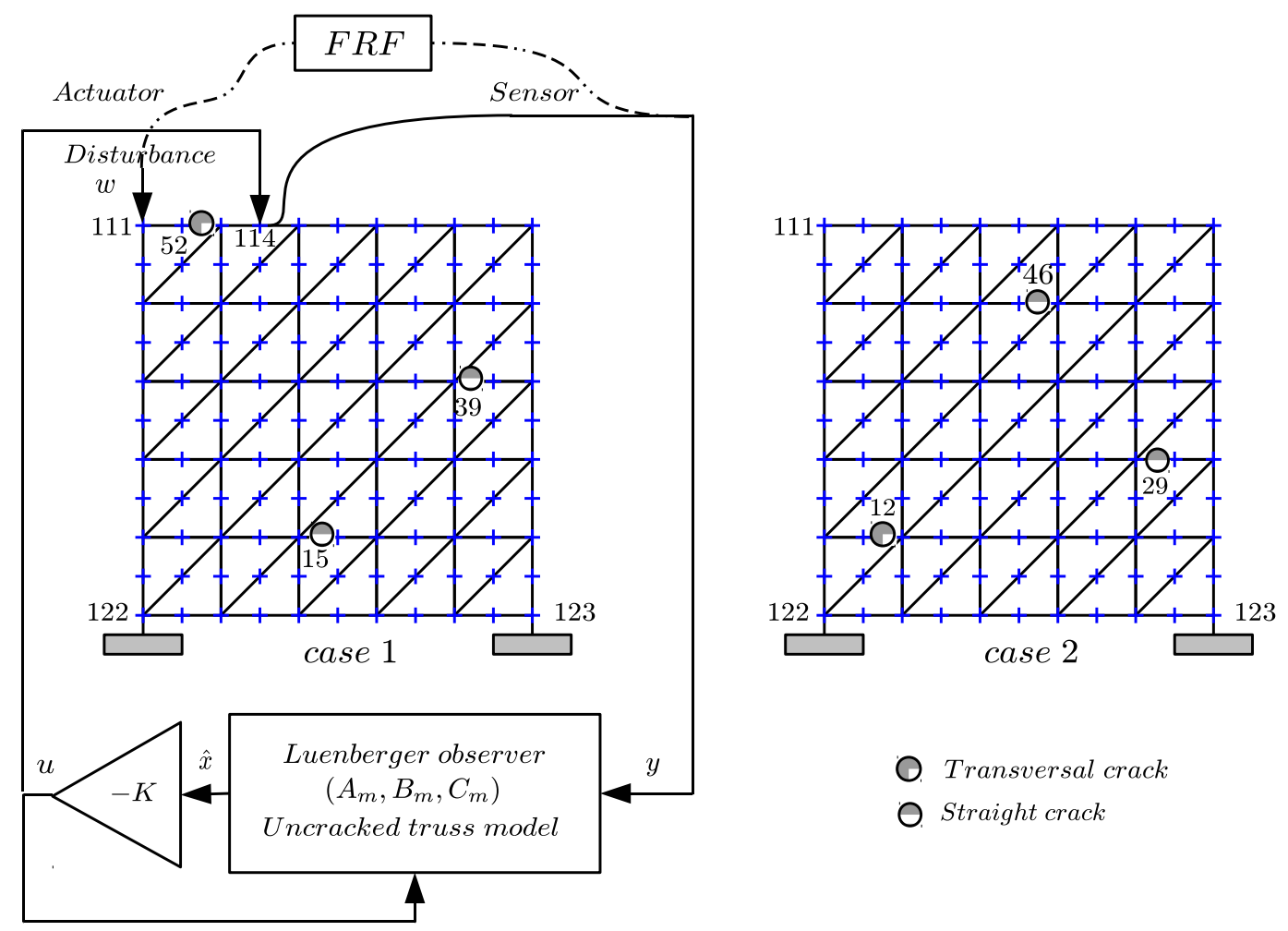

Figure 2: The control loop using the Luenberger observer applied to the multi-cracked truss structure and the FRF measurement points in two cracks cases (-: control loop, -.-: FRF measurements between disturbance and sensor points)

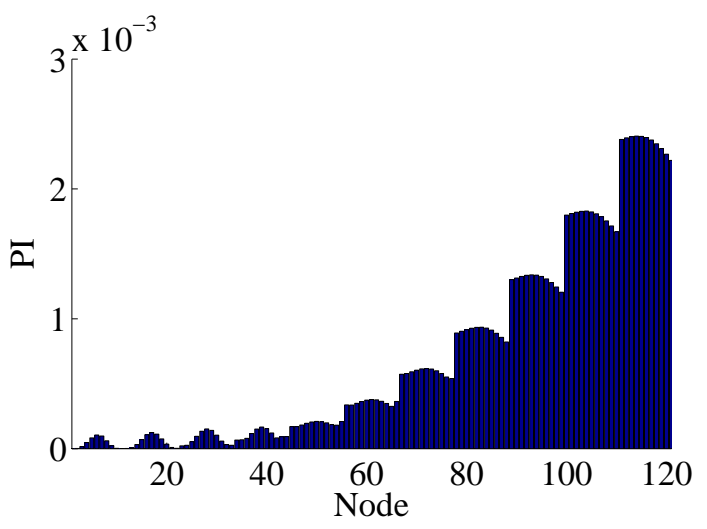

(a) Hac and Liu criterion calculated on the truss

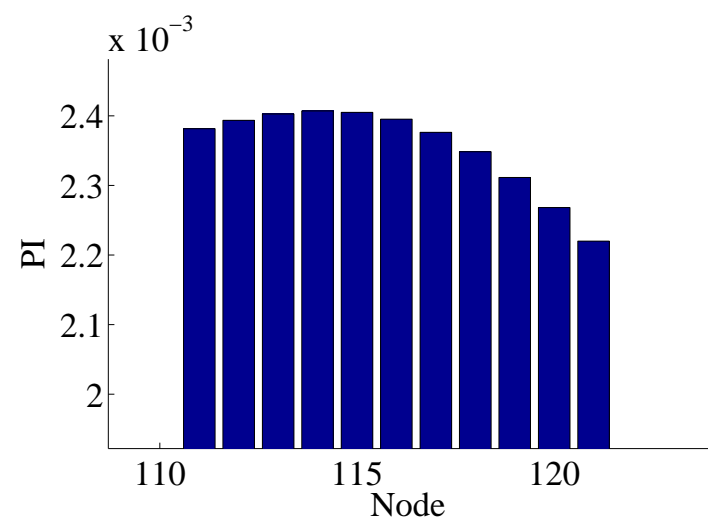

(b) Zoom on nodes 111 to 121

Figure 3: Optimal placement for the control of the truss

observed: frequency increases with crack closure (Sinou 2007).

Moreover, the orientation of the crack causes a damping variation $(-0.4 \%$ and $-0.5 \%$ for the first mode, $-0.3 \%$ and $-1.5 \%$ for the second mode, $-6.7 \%$ and $-7.3 \%$ for the third mode, $5.1 \%$ and $5.1 \%$ for the fourth mode, $0.5 \%$ and $0.5 \%$ for the fifth mode and $0.3 \%$ and $0.3 \%$ for the sixth mode in the cases 1 and 2 respectively). Consequently, damping increases or decreases with crack closure depends on the mode. Finally, the crack orientation causes a variation of modal amplitude $(-1.2 \%$ and $-1.2 \%$ for the first mode, $-0.3 \%$ and $-0.3 \%$ for the second mode, 

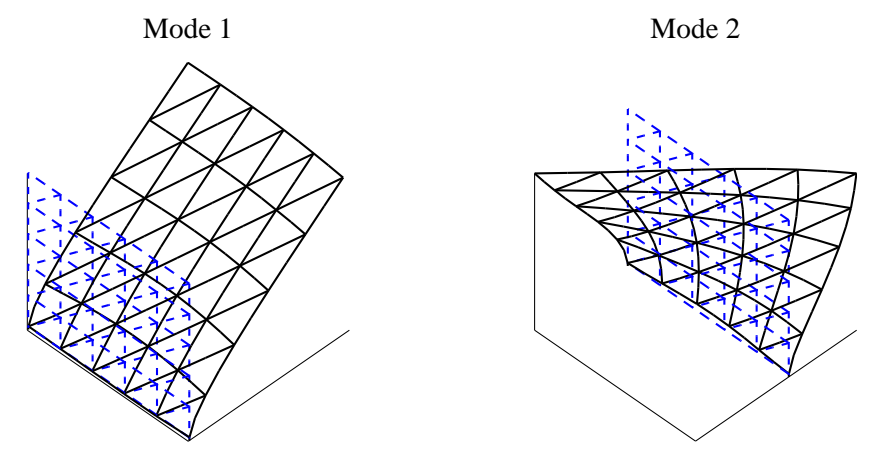

Mode 4

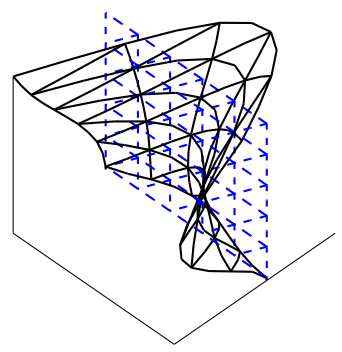

Mode 5

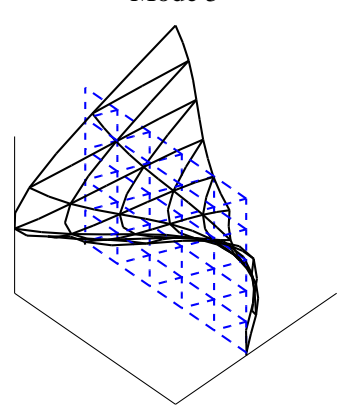

Mode 6

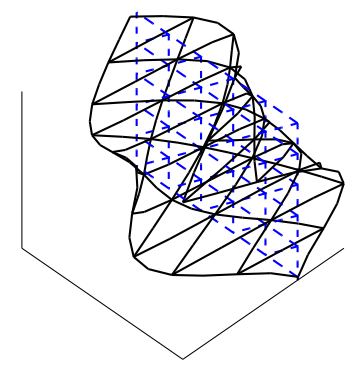

Figure 4: Mode shapes of the uncracked truss

$-0.6 \%$ and $-0.6 \%$ for the third mode, $-0.2 \%$ and $-0.1 \%$ for the fourth mode, $-0.7 \%$ and $-0.6 \%$ for the fifth mode and $-4.1 \%$ and $-2.1 \%$ for the sixth mode in the cases 1 and 2 respectively). Consequently, modal amplitude decreases with crack closure. These first results indicated that a crack can be detected by estimating modal parameters.

\subsection{Multi-cracked controlled truss}

Due to the effects of cracks that have been observed in the previous section, impacts of the variations on a multi-cracked controlled truss are undertaken. Indeed, in the case of controlled structure, the damping and amplitude variation can modify the controller efficiency. That is why these variations have to be identified.

First of all, estimated modal parameters of the nominal uncracked controlled truss are detailed in table 2. Then, the incidence of crack orientation on the controlled structure is shown in figure $7(\mathrm{a})$ to figure $7(\mathrm{f})$ in the first case and in figure $8(\mathrm{a})$ to figure $8(\mathrm{f})$ in the second case for each mode. The references, i.e. the uncontrolled and controlled FRF of the uncracked truss corresponding to the nominal configuration, are shown in solid and dotted black lines respectively. The LQ gain $\mathbf{K}$ and the LQG gain $\mathbf{L}$ are obtained by using the model $\left(\mathbf{A}_{m}, \mathbf{B}_{m}, \mathbf{C}_{m}\right)$ of the uncracked truss and are not modified when the crack orientation $\chi$ is changed. Consequently, for each crack orientation, there are some differences between the real structure represented by the system $\left(\mathbf{A}_{\chi}, \mathbf{B}_{\chi}, \mathbf{C}_{\chi}\right)$ and the observer model $\left(\mathbf{A}_{m}, \mathbf{B}_{m}, \mathbf{C}_{m}\right)$. These differences cause variations in control performance and underline variation of crack orientations in the case where estimating the variation modal parameters is difficult. 

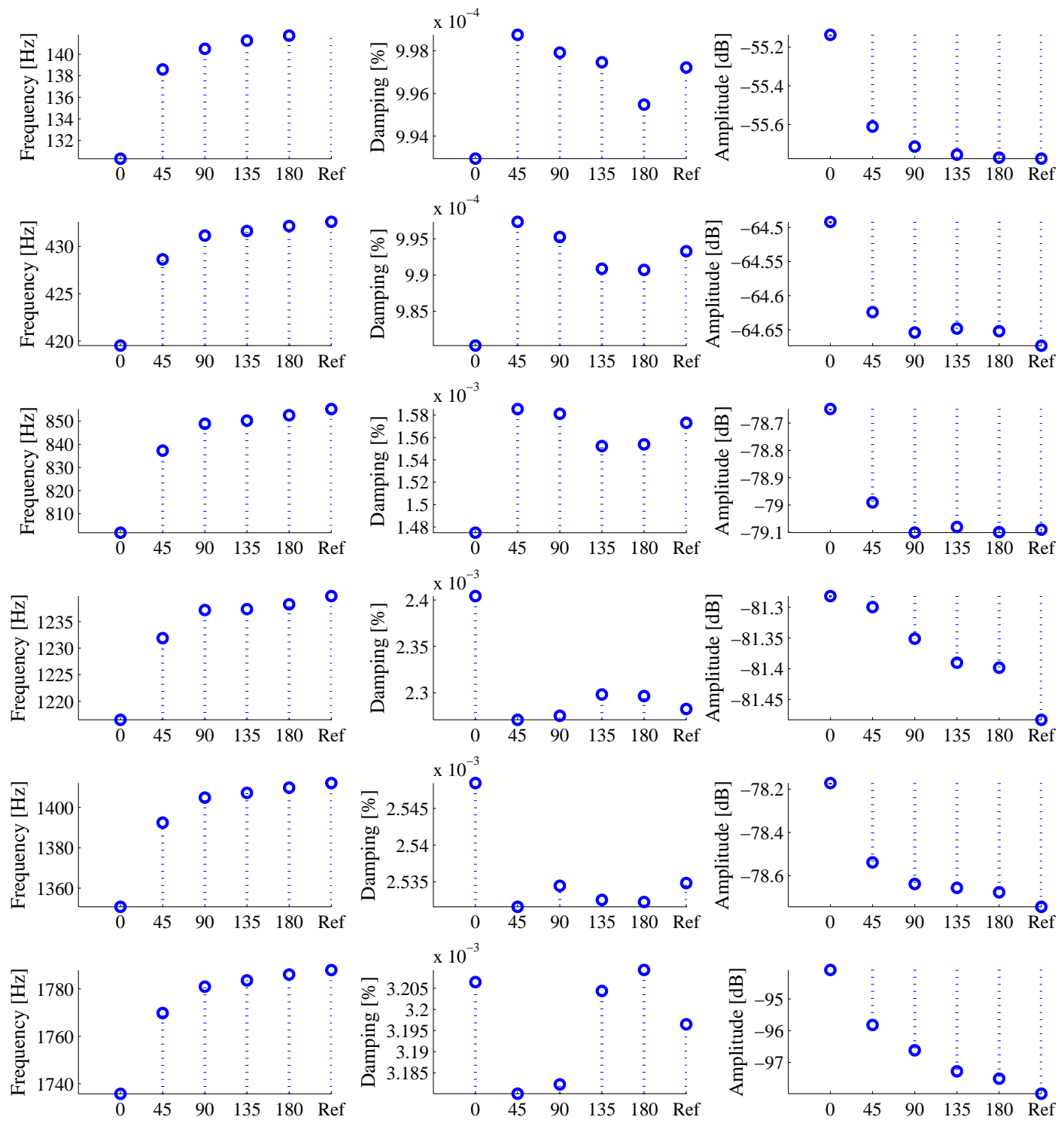

Figure 5: Estimated modal parameters (frequency, damping and amplitude) for $\chi=0^{\circ}$ to $\chi=180^{\circ}$ in the case 1 and uncracked modal parameters (ref.)

For example, we consider the evolution of the cracked orientation from $\chi=0^{\circ}$ to $\chi=180^{\circ}$ for the transversal crack and the two straight cracks on the controlled truss. The effects on the modal parameters are illustrated in figures 9 and 10 for the two cases. The crack orientation from $\chi=0^{\circ}$ to $\chi=180^{\circ}$ causes a frequency variation $(-11,2 \%$ for the first mode, $-4,0 \%$ for the second mode, $-7,4 \%$ for the third mode, $-2,0 \%$ for the fourth mode, $-5,3 \%$ for the fifth mode and $-6,9 \%$ for the sixth mode in the case $1 ;-11,1 \%$ for the first mode, $-4,1 \%$ for the second mode, $-7,1 \%$ for the third mode, $-2,6 \%$ for the fourth mode, $-5,2 \%$ for the fifth mode and $-3,8 \%$ for the sixth mode in the case 2 ). Consequently, controlled system frequencies 

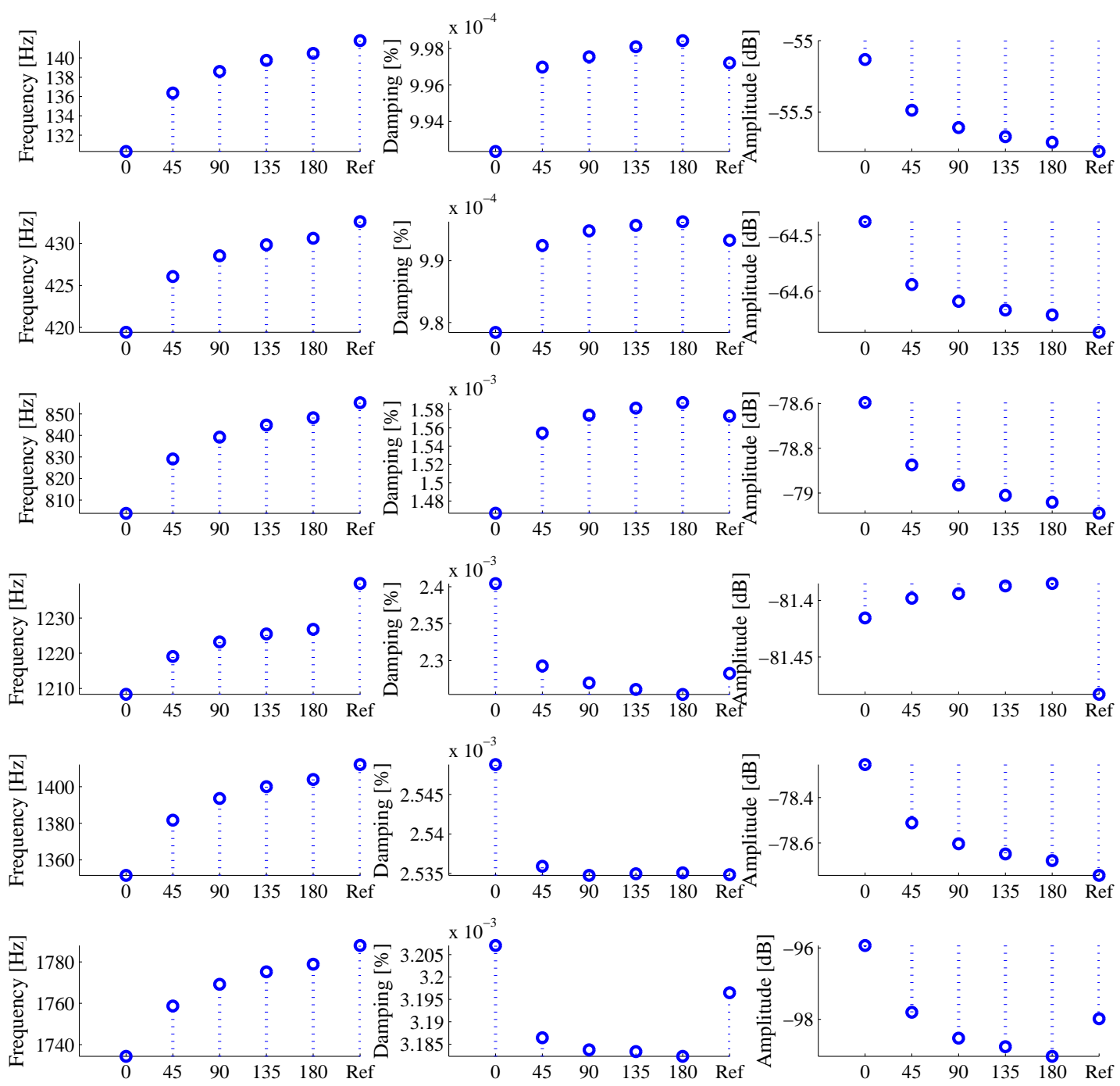

Figure 6: Estimated modal parameters (frequency, damping and amplitude) for $\chi=0^{\circ}$ to $\chi=180^{\circ}$ in the case 2 and uncracked modal parameters (ref.)

\begin{tabular}{ccc}
\hline Frequency $[\mathrm{Hz}]$ & Modal damping & Modal amplitude $[\mathrm{dB}]$ \\
\hline 141,1 & 0,136 & $-76,28$ \\
431,8 & 0,029 & $-78,55$ \\
850,8 & 0,011 & $-86,18$ \\
1235,2 & 0,003 & $-83,09$ \\
1407,5 & 0,012 & $-84,35$ \\
1496,5 & 0,029 & $-99,60$ \\
\hline
\end{tabular}

Table 2: Modal parameters of the uncracked controlled truss

increase with crack closure. Due to the control performances modification, the orientation of the crack causes a very large damping variation on the controlled truss $(9,7 \%$ and $9,9 \%$ for 


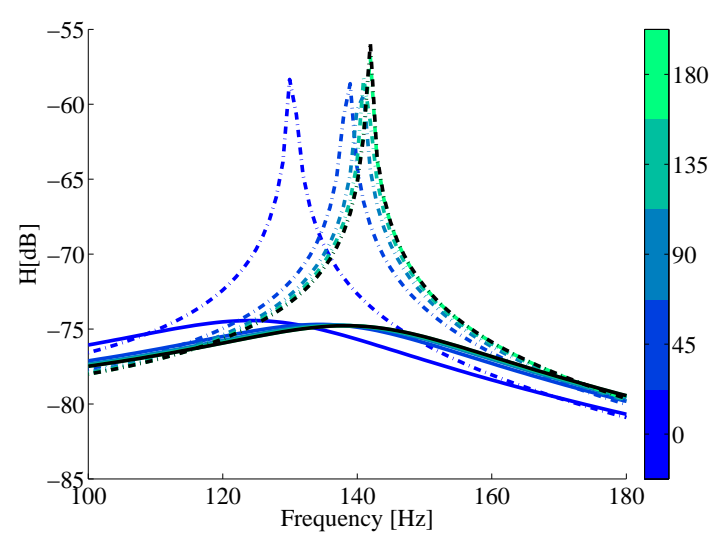

(a) First mode

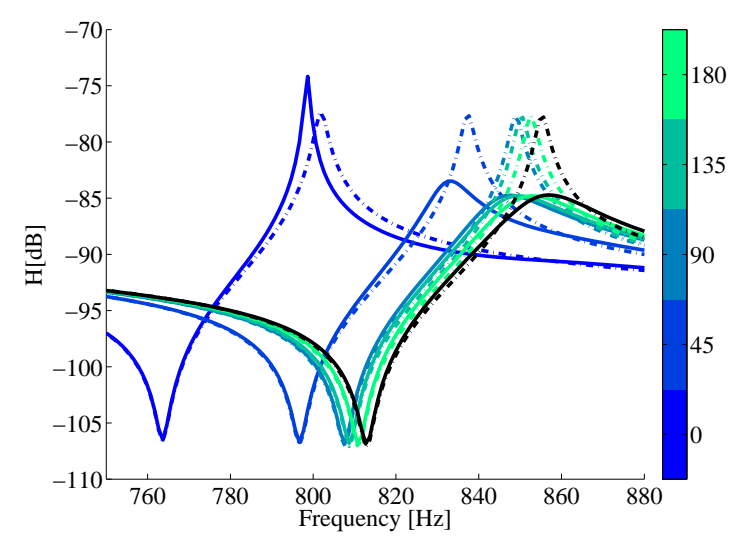

(c) Third mode

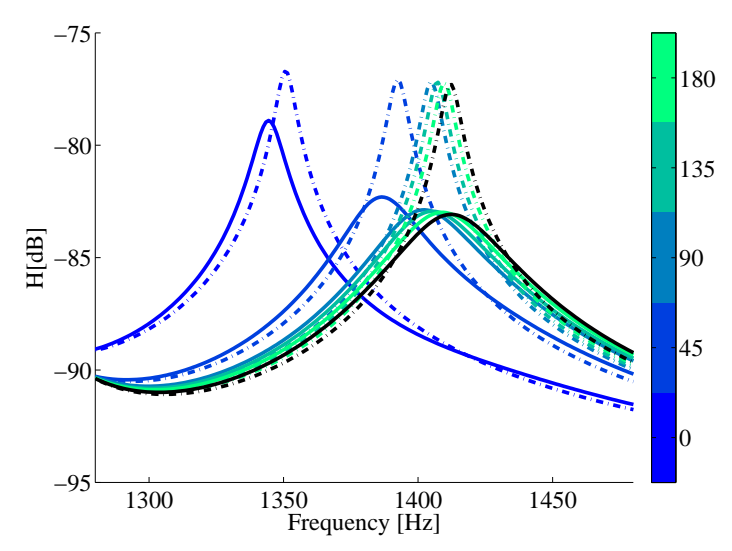

(e) Fifth mode

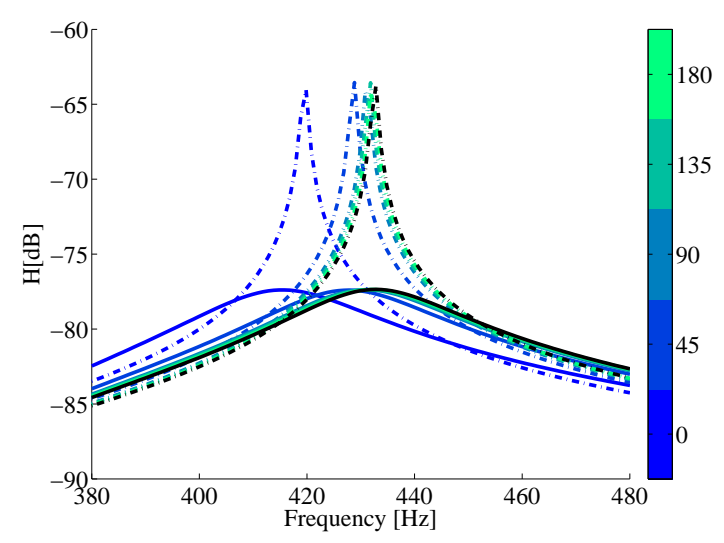

(b) Second mode

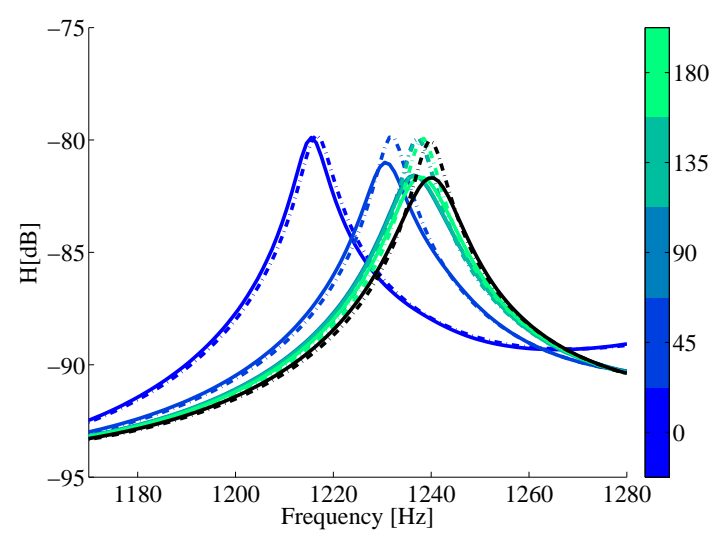

(d) Fourth mode

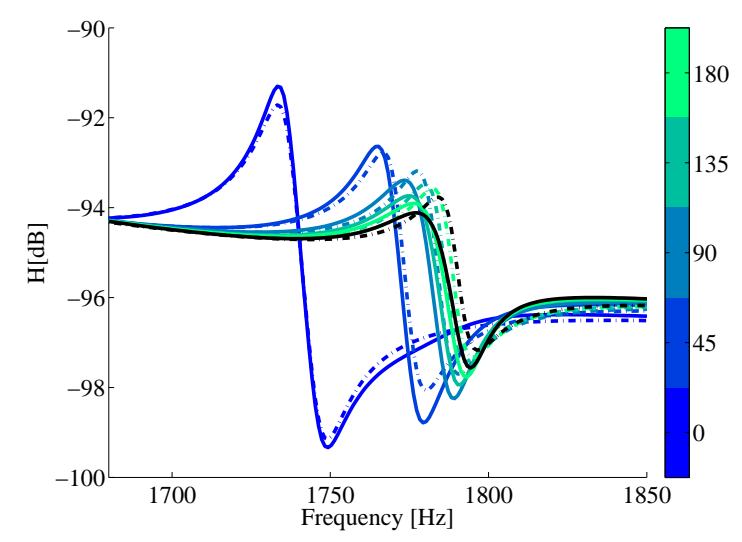

(f) Sixth mode

Figure 7: Influence of a transversal crack on a multi-cracked controlled truss for $\chi=0^{\circ}$ to $\chi=180^{\circ}$ in the case 1 and uncracked reference in black line (-.-: uncontrolled truss, controlled truss)

the first mode, $-0,6 \%$ and $1,0 \%$ for the second mode, $-807,9 \%$ and $-620,9 \%$ for the third mode, $-141,8 \%$ and $-154,9 \%$ for the fourth mode, $-91,8 \%$ and $-112,9 \%$ for the fifth mode and $-48,4 \%$ and $-217,5 \%$ for the sixth mode in the case 1 and 2 respectively). Finally, the crack orientation causes a variation of modal amplitude $(-0,47 \%$ and $-0,5 \%$ for the first mode, $-0,12 \%$ and $-0,1 \%$ for the second mode, $-12,4 \%$ and $-11,2 \%$ for the third mode, $-2,9 \%$ and $-2,8 \%$ for the fourth mode, $-3,8 \%$ and $-4,0 \%$ for the fifth mode and $-3,6 \%$ and $-4,0 \%$ for the sixth mode in the case 


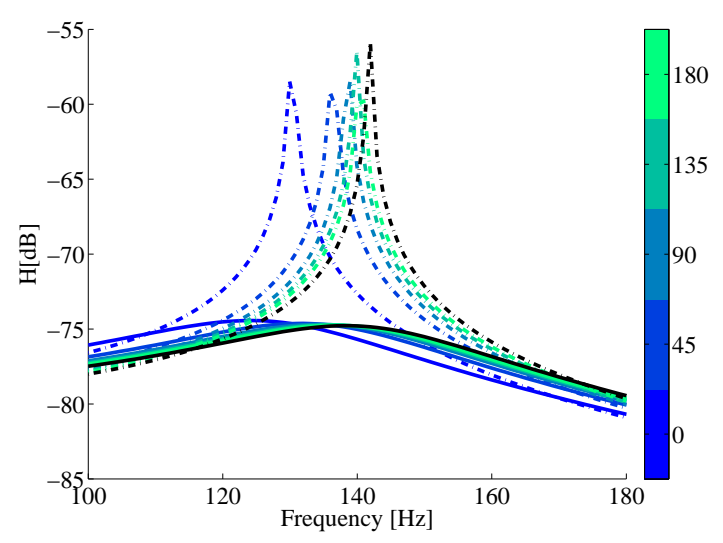

(a) First mode

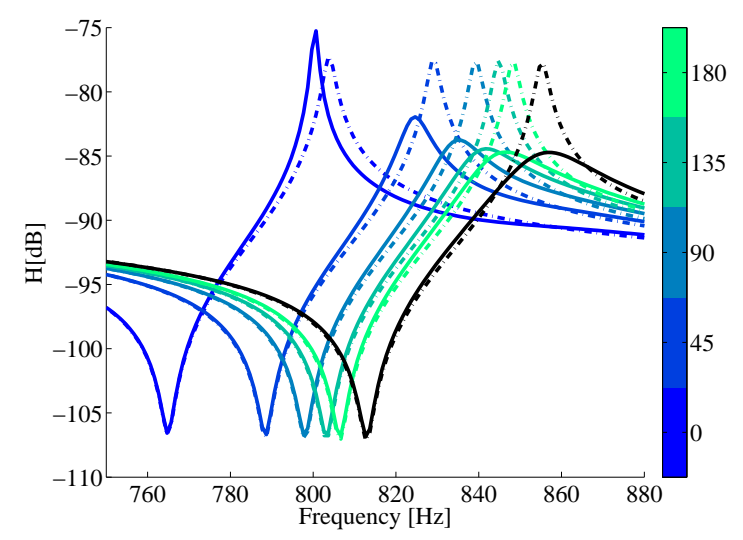

(c) Third mode

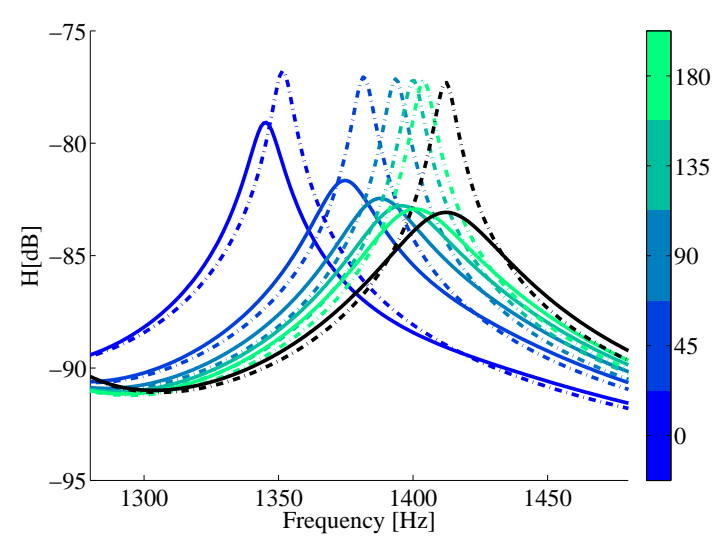

(e) Fifth mode

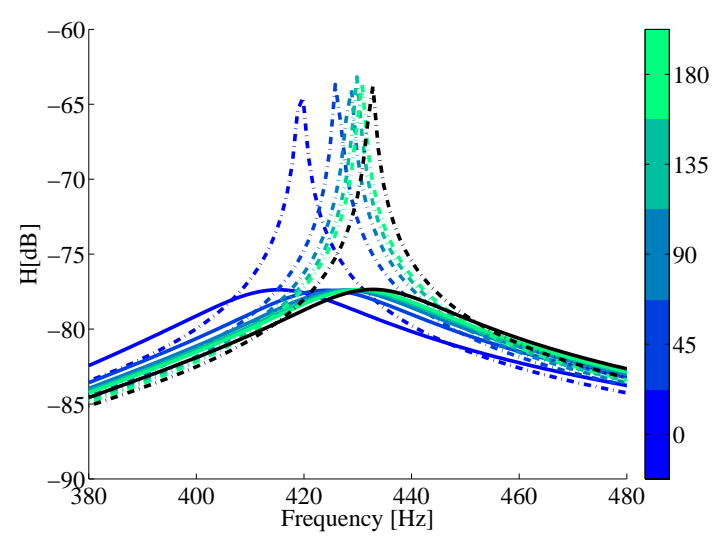

(b) Second mode

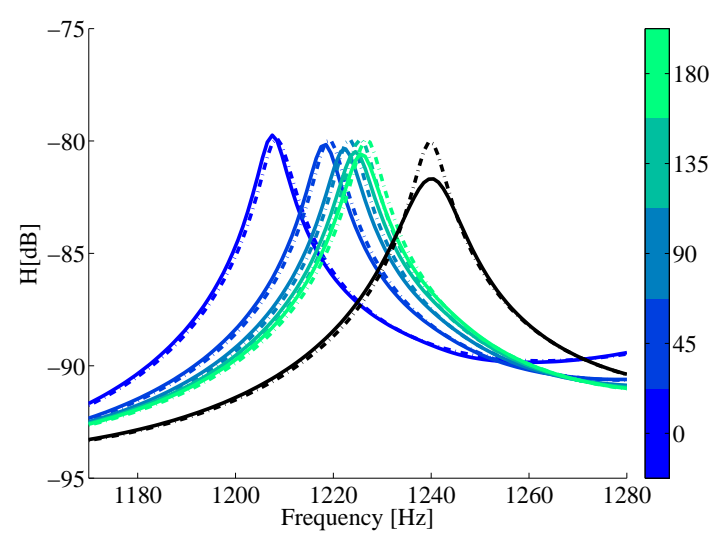

(d) Fourth mode

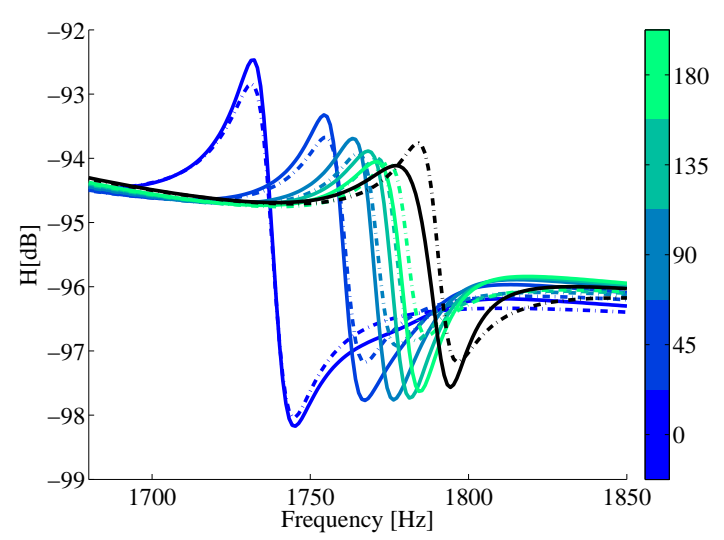

(f) Sixth mode

Figure 8: Influence of a transversal crack on a multi-cracked controlled truss for $\chi=0^{\circ}$ to $\chi=180^{\circ}$ in the case 2 and uncracked reference in black line (-.-: uncontrolled truss, controlled truss)

1 and 2 respectively).

For the reader comprehension, in the case of controlled truss, the variations due to the crack orientation in the frequency response function result from structural truss modification and from changes in the controller dynamics. Indeed the variation of frequencies and mode shapes induce some differences between the modal matrices $A_{m}$ and $B_{m}$ of the uncracked truss used in the Luenberger observer, and the modal matrices $A_{s}$ and $B_{s}$ of the cracked truss. These 
differences modify the controller dynamics and cause a large variation of the active damping induced by the control. Also a large variation of magnitude on the controlled frequency response function can be observed, particularly for the third and fifth modes. In the case of uncontrolled truss, the variations due to the crack orientation in the frequency response function result only of structural truss modifications.

These results demonstrate that crack orientation causes large changes in control performance depends on the mode, that can be positive or negative. These variations could be used as an indicator of crack orientation variation by undertaking links between the crack orientation and control reduction variation in future works.
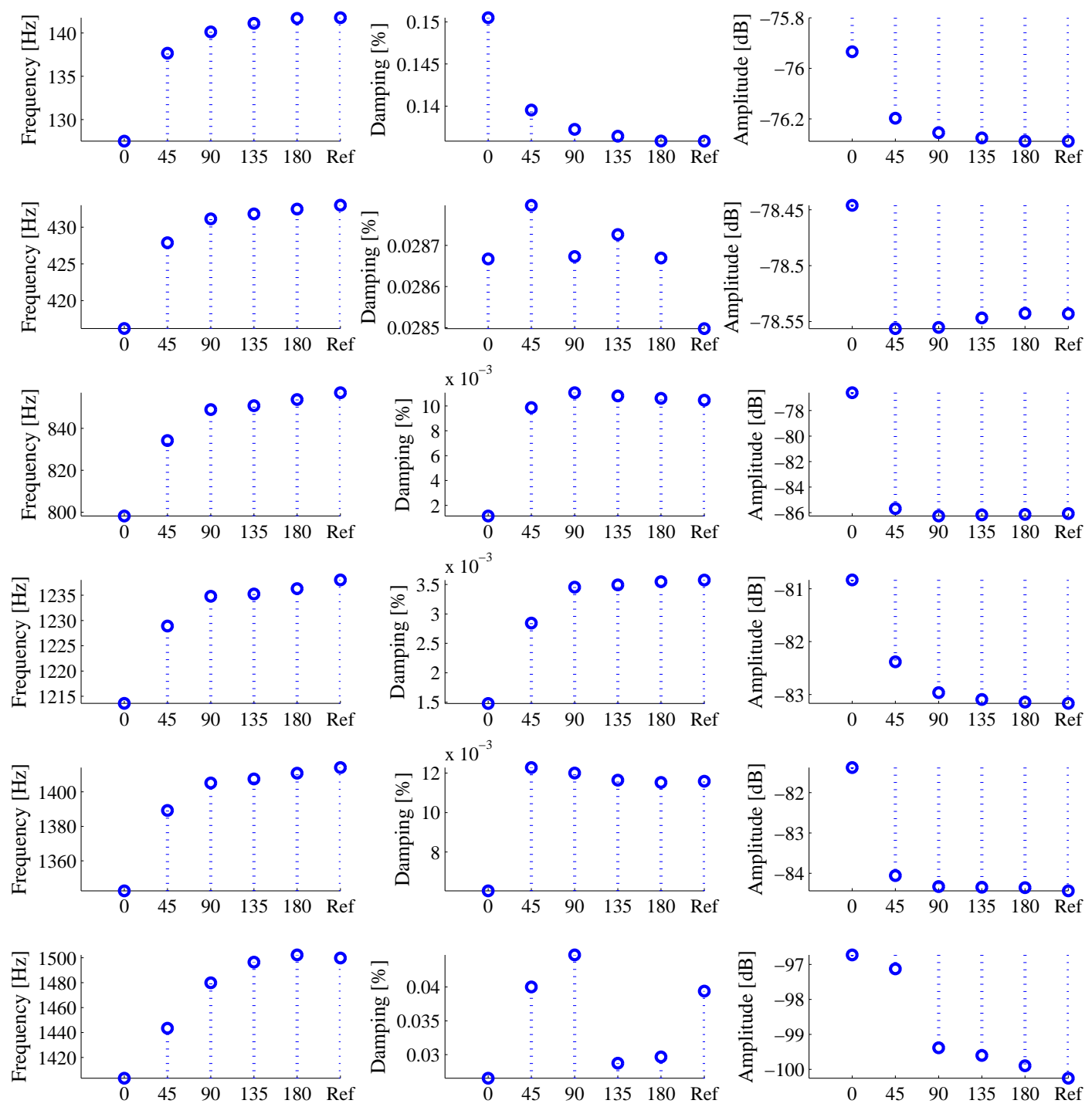

Figure 9: Estimated modal parameters (frequency, damping and amplitude) for the controlled truss for $\chi=0^{\circ}$ to $\chi=180^{\circ}$ in the case 1 and uncracked modal parameters (ref.) 

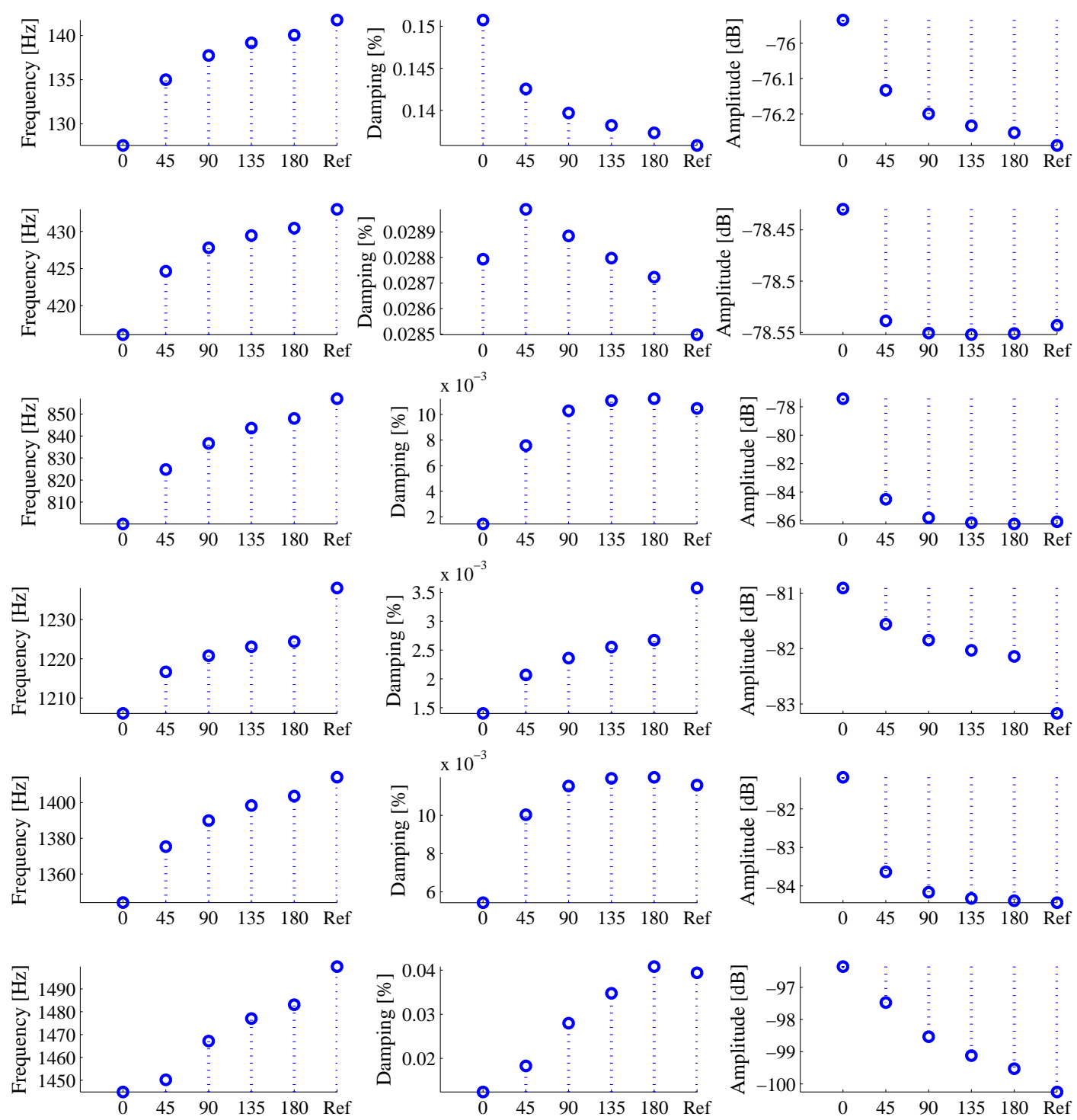

Figure 10: Estimated modal parameters (frequency, damping and amplitude) for the controlled truss for $\chi=0^{\circ}$ to $\chi=180^{\circ}$ in the case 2 and uncracked modal parameters (ref.)

\section{Conclusion}

This study proposes an investigation into the efficiency of an optimal control and identification procedure for a complex structure with multiple cracks. This method is applied numerically to a multi-cracked controlled truss by considering not only evolutions of crack depth and position but also crack orientation, which can drastically modify the dynamic behavior of damaged mechanical structures. Both the optimal control of the cracked structure and the possibility of detecting the presence of cracks by monitoring the evolution of control performance are studied. It is shown that the evolution of control parameters and modal parameters (particu- 
larly modal damping) of the controlled system in relation to other parameters (such as crack orientation) can be indicators of the presence of a crack in a mechanical structure. Especially, it is observed that the reduction in decibels obtained with the control of the multi-cracked system can be a way to predict the appearance of crack. Finally, this approach could be the first step of a health monitoring strategy in order to reduce vibration in a cracked structure. Future works should be linked between the crack orientation and control reduction variation.

\section{Acknowledgments}

The authors gratefully acknowledge the financial support of the French National Research Agency through the Young Researcher program ANR-07-JCJC-0059-01-CSD2.

\section{References}

Badel A, Guyomar D, Lefeuvre E and Richard C (2006) Piezoelectric energy harvesting using a synchronized switch technique. Journal of Intelligent Material and Structures 17(8-9): 831-839.

Chomette B, Chesne S, Remond D, and Gaudiller L (2010) Damage reduction of on-board structures using piezoelectric components and active modal control - application to a printed circuit board. Mechanical System and Signal Processing 24(2): 390-397.

Chomette B, Remond D, Chesne S and Gaudiller L (2008) Semi-adaptive modal control of on-board electronic boards using identification method. Smart Material and Structures 17(6): $1-8$.

Davies WG and Mayes IW (1984) The vibrational behaviour of a multi-shaft, multi-bearing system in the presence of a propagating transverse crack. Transactions of the ASME Journal of Vibration, Acoustics, Stress, and Reliability in Design 106: 146-153.

Dilena M and Morassi A (2002) Identification of crack location in vibrating beams from changes in node positions. Journal of Sound and Vibration 255(5): 915-930.

Doebling S, Farrar C, Prime M and Shevitz D (1996) Damage Identification and Health Monitoring of Structural and Mechanical Systems from Changes in Their Vibration Characteristics: A Literature Review. Technical Report LA-13070-MS: Los Alamos National Laboratory Report.

Faverjon B and Sinou J-J (2008) Robust damage assessment of multiple cracks based on the frequency response function and the constitutive relation error updating method. Journal of Sound and Vibration 312(4-5): 821-837.

Faverjon B and Sinou J-J (2009) Identification of an open crack in a beam by using a posteriori error estimator on the frequency response functions with noisy measurements. European Journal of Mechanics A-Solid 28(1): 75-85.

Gaudiller L and Der Hagopian J (1996) Active control of flexible structures using a minimum number of components. Journal of Sound and Vibration 193(3): 713-741.

Hac A and Liu L (1993) Sensor and actuator location in motion control of flexibles structures. 
Journal of Sound and Vibration 137(2): 239-261.

Lees AW and Friswell M (2001) The vibration signature of chordal cracks in asymmetric rotors. Proceedings of the 19th International Modal Analysis Conference: 1-6.

Luenberger D (1971) An introduction to observers. Automatic Control, IEEE Transactions on 16(6): 596-602.

Preumont A (2002) Vibration Control of Active Structures: An introduction, second ed.. Dordrecht: Springer, Kluver Academic.

Richardson Mark H and Formenti DL (1985) Global curve fitting frequency response measurements using the rational fraction polynomial method. Proceeding of the International Modal Analysis Conference and Exhibit 1: 390-397.

Salawu O S (1997) Detection of structural damage through changes in frequency: a review. Engineering Structures 19(9): 718-723.

Sinou J-J (2007) Damage Assessment Based on the Frequencies' Ratio Surfaces Intersection Method for the Identification of the Crack Depth, Location and Orientation. Structural Durability and Health Monitoring 3(3): 134-162.

Sinou J-J and Lees AW (2005) The influence of cracks in rotatingshafts. Journal of Sound and Vibration 285(4-5): 1015-1037.

Sinou J-J (2009) A review of damage detection and health monitoring of mechanical systems from changes in the measurement of linear and non-linear vibrations. In: Mechanical Vibrations: Measurement, Effects and Control. Nova Science Publishers: Robert R. Sapri, ISBN 978-1-60692-037-4.

Sohn H, Farrar C, Hemez F, Shunk D, Stinemates D and Nadler B (2003) A Review of Structural Health Monitoring Literature: 1996-2001. Technical Report LA-13976-MS: Los Alamos National Laboratory Report. 